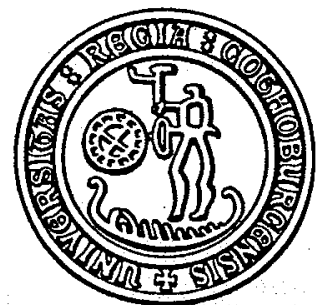

Research Report Department of Statistics

Göteborg University

Sweden

\title{
Detection of turning points in business cycles
}

\author{
Eva Andersson \\ David Bock \\ Marianne Frisén
}

Mailing address: Dept of Statistics P.O. Box 660 SE 40530 Göteborg Sweden

\section{Fax}

Nat: 031-773 1274

Int: +46317731274

\section{Phone}

Nat: 031-773 1000

Int: +46317731000
Home Page:

http://www.stat.gu.se/stat 


\title{
Detection of Turning Points in Business Cycles
}

\author{
Eva Andersson
}

David Bock

Marianne Frisén

\begin{abstract}
Methods for on-line monitoring of business cycles are compared with respect to the ability of early prediction of the next turn by an alarm for a turn in a leading index. Three likelihood based methods for turning point detection are compared in detail by using the theory of statistical surveillance and by simulations. One of the methods is based on a Hidden Markov Model. Another includes a non-parametric estimation procedure. Evaluations are made of several features such as knowledge of shape and parameters of the curve, types and probabilities of transitions and smoothing. Results on the expected delay time to a correct alarm and the predictive value of an alarm are discussed. The three methods are also used to analyze an actual data set of a period of the Swedish industrial production. The relative merits of evaluation of methods by one real data set or by simulations are discussed.
\end{abstract}

Key Words: Business cycle; Early warning; Monitoring; Optimal; Likelihood ratio; Bayes; Markov; HMM; Switching regime; Turning point; Non-parametric

JEL Classification: C14, C15, C44, E32 
Leading economic indicators can be used to predict the turns of the business cycles. The turning point time of the general business cycle can be predicted by timely detection of the turns in a leading indicator. In this paper, predictions of the actual value of the business cycle are not discussed. Instead the methods discussed here are concerns with detecting the turning point time, i.e. a change from a recession phase to an expansion phase (or vice versa). Predictions of the turning point times can be made by using information from one or several time series, which are leading in relation to the actual business cycle. By applying a system for detecting turning points in a leading indicator we can receive early signals about the future behavior of the business cycle. For reviews and general discussions see e.g. Neftci (1982), Zarnowitz and Moore (1982), Westlund and Zackrisson (1986), Hackl and Westlund (1989), Zellner et al. (1991), Jun and Joo (1993), Lahiri and Wang (1994), Li and Dorfman (1996) and Birchenhall et al. (1999).

Methods based on likelihood or posterior distributions have been in focus in recent years. In the general theory on statistical surveillance there are proofs for their optimality properties (see e.g. Shiryaev (1963) and Frisén and de Maré (1991)). Many of the suggested methods are based on a hidden Markov model (HMM).

In a surveillance situation repeated decisions are made, which is important to consider when the inference is made. Continual observation is made of a time series with the goal of detecting the turning point in the underlying process as soon as possible. For general reviews on statistical surveillance, see Shiryaev (1963), Frisén and de Maré (1991), Wetherhill and Brown (1991), Srivastava and Wu (1993), Lai (1995), Frisén and Wessman (1999) and Frisén (2002).

In this paper some differences and similarities between suggested likelihood based methods for turning point detection in cyclical, economic processes are shortly described. Furthermore, there is a short description of how the methods perform when used to analyze an actual data set of a period of the Swedish industrial production. For details see Andersson et al. (2002a) and Andersson et al. (2002b).

The paper is organized as follows. Section 2 contains a description of different likelihood based approaches. In Section 3 the results of the Monte Carlo study conducted in Andersson et al. (2002b) in order to compare the performance of three different likelihood based methods are briefly summarized. In Section 4 the three methods are used to analyze a period of the Swedish industrial production and the pros and cons of this way to evaluate methods is discussed. Section 5 contains a summarizing discussion. 


\section{$2 \quad$ Likelihood based surveillance for detection of turning points}

\subsection{Model for each state}

The variable under surveillance, $X$, is a leading economic indicator. By monitoring $X$ we want to detect a regime shift (a turning point) as soon as possible. The model for $X$ discussed here is:

$$
X(t)=\mu(t)+\varepsilon(t)
$$

where $\varepsilon(t) \sim \mathrm{iid} \mathrm{N}\left[0 ; \sigma^{2}\right]$ and $\mu(t)$ is a stochastic process.

The aim is to detect the change in $\mu$, from expansion state to recession state (or vice versa). The definition of a turning point is that the regression of $\mu$ on time is monotonic within each regime:

$$
\mathrm{E}\left[\mathrm{X}_{\mathrm{t}}\right]=\mu_{t}=\left\{\begin{array}{lr}
\mu(1) \leq \mu(2) \leq \ldots \leq \mu(t), & t<\tau \\
\mu(1) \leq \ldots \leq \mu(\tau-1) \text { and } \mu(\tau-1) \geq \ldots \geq \mu(t), & t \geq \tau
\end{array}\right.
$$

where $t=1$ is in a period of expansion and $\tau$ is the turning point from the expansion to a recession. The turning point time, $\tau$, is stochastic and hence $\mu$ is stochastic.

Often a parametric assumption is used, for example that the regression is linear within each phase

$$
\mathrm{E}[\mathrm{X}(\mathrm{t})]=\mu(t)=\left\{\begin{array}{ll}
\beta_{0}+\beta_{1} \cdot t, & t<\tau \\
\beta_{0}+\beta_{1} \cdot(\tau-1)-\beta_{2} \cdot(t-\tau+1), & t \geq \tau
\end{array},\right.
$$

where $t=\{1,2, \ldots\}$. A consequence is that the expected value of the differentiated process is assumed to be constant, conditional on the state:

$$
\mathrm{E}[\mathrm{X}(\mathrm{t})-\mathrm{X}(\mathrm{t}-1)]= \begin{cases}\beta_{1}, & t<\tau \\ \beta_{2}, & t \geq \tau\end{cases}
$$

where $\beta_{1}$ and $\beta_{2}$ often are assumed known.

In addition to the assumption of linearity within phases it is sometimes assumed that the slopes are symmetrical for the two phases $\left(\beta_{1}=\beta_{2}\right)$.

The standard deviation $\sigma$ can be assumed to be different for recession and expansion. Here, the variance is assumed equal, as also in Andersson (2001). 


\subsection{Specifications of the events to be detected}

The unknown time when the underlying process $\mu$ changes from expansion to recession (a peak), or vice versa is denoted $\tau$. At decision time $s$ an alarm statistic is used to discriminate between $D(s)=\{\tau>s\}$ and $C(s)=\{\tau \leq s\}$. Knowledge of whether the next turn will be a trough or a peak is assumed. For a method that relies only on the assumption of monotonicity restrictions, the aim is to discriminate between the following two events:

$$
\begin{aligned}
& D(s): \mu(1) \leq \ldots \leq \mu(s) \\
& C(s): \mu(1) \leq \ldots \leq \mu(\tau-1) \text { and } \mu(\tau-1) \geq \mu(\tau) \geq . . \geq \mu(s)
\end{aligned}
$$

where $\tau=\{1,2, \ldots, s\}$ and at least one inequality is strict in the second part.

If an additional assumption is made, namely that the regression consist of linear functions where the slopes are symmetrical for the two phases, then the aim is to discriminate between $D$ and $C$, such that

$$
\begin{aligned}
& D(s): \mu(s)=\beta_{0}+\beta_{1} \cdot s \\
& C(s)=\{\cup C(\tau)\},
\end{aligned}
$$

where $C(\tau): \mu(s)=\beta_{0}+\beta_{1} \cdot(\tau-1)-\beta_{1} \cdot(s-\tau+1)$ and where $\tau=\{1,2, \ldots, s\}$ and $\beta_{0}$ and $\beta_{1}$ are known constants.

If a HMM is assumed, the situation is such that at decision time $s$ an alarm statistic is used to discriminate between

$$
\begin{aligned}
& D(s): \mu(s-1) \leq \mu(s), \\
& C(s): \mu(s-1)>\mu(s) .
\end{aligned}
$$

When comparing (5) and (6) the difference between $D$ and $C$ is only the assumptions on $\mu(t)$. However, when a HMM is used, as in (7), the events are different also in another aspect. The apparently simpler event in the HMM approach is combined with a more complicated situation for the information of previous states. No knowledge of previous states is utilized in a HMM approach. Thus the events in (7) correspond to families of histories of states. Because of Markov dependence, the earlier observations carry information of the history of states. 


\subsection{Assumptions regarding transition probabilities}

The common assumption of a time invariant transition probabilities between the states is made for all three methods investigated here.

When the information about the time and type (peak or trough) of the last turning point is utilized, as in (5) and (6) and Neftci (1982), it is sufficient with the single transition probability

$$
v=P(C(t) \mid D(t-1))=P(\tau=t \mid \tau \geq t) .
$$

When the information about the time and type of the last turning point cannot be used, it is necessary to make a probability statement regarding the type of the next turning point as well as inference about whether the turning point has occurred. For this purpose, it is necessary to consider all previous possible turns. Therefore, two intensities are needed. The intensity parameters can be given as transition probabilities from expansion state to recession state and vice versa.

One approach which will be described below is to use a non-informative prior for the turning point time.

\subsection{Alarm statistics and alarm limits}

The full likelihood ratio method (LR) has several optimality properties, see Frisén and de Maré (1991). The likelihood ratio method yields a minimum expected delay of an alarm for a fixed probability of false alarm. The full likelihood ratio method signals an alarm for the first time $s$ for which

$$
\mathrm{LR}(s)=\frac{f\left(x_{s} \mid C\right)}{f\left(x_{s} \mid D\right)}>k_{\mathrm{s}},
$$

where $f$ is the likelihood function and $k_{s}=(k /(1-k) \cdot(P(D(s)) / P(C(s)))$.

For the situation where $C$ is the complement of $D$, Frisén and de Maré (1991) demonstrated that the posterior probability approach is equivalent to the likelihood ratio approach. All methods considered here use a likelihood ratio based alarm statistic.

The full likelihood ratio requires an assumption about $\tau$. When no reliable information is available a non-informative prior can be used. This is sometimes named the Shiryaev-Roberts (SR) approach. 
For the situation where $\mu(\mathrm{t})$ is modeled using linear functions with a symmetric turning point (see (6)) and where the SR approach is used, the alarm rule for the likelihood ratio at time $s$ is written as

$$
\sum_{t=1}^{s} \frac{f\left(x_{s} \mid \mu=\mu^{C t}\right)}{f\left(x_{s} \mid \mu=\mu^{D}\right)}>k_{1}
$$

where $k_{1}$ is a constant alarm limit. This method is hereafter referred to as the SRlin method.

Frisén (1994) suggested a method where no knowledge of $\mu$ is assumed, other than the monotonicity restrictions in (5), and where the SR approach regarding the intensity is used. This method was evaluated by Andersson (2002) and Andersson et al. (2002b). The alarm rule is

$$
\sum_{t=1}^{s} \frac{f\left(x_{s} \mid \mu=\hat{\mu}^{C t}\right)}{f\left(x_{s} \mid \mu=\hat{\mu}^{D}\right)}>k_{2},
$$

where $\mathrm{k}_{2}$ is a constant alarm limit, $\hat{\mu}^{D}$ is the maximum likelihood estimator of the vector $\mu$ under the monotonicity restriction $D$ (see e.g. Robertson et al. (1988)) and $\hat{\mu}^{c t}$ is the maximum likelihood estimator of the vector $\mu$ under the monotonicity restriction $C t$ (see Frisén (1986)). This method is hereafter referred to as the MSR method.

In Hidden Markov Modeling (HMM) approaches, e.g. Neftci (1982), Hamilton (1989), Layton (1996), Koskinen and Öller (1998) and Kim and Nelson (1998) the posterior probability is used as the alarm statistic. Thus the method signals an alarm as soon as

$$
P\left(C(s) \mid x_{s}\right)>c,
$$

where $c$ often is choosen to 0.5 . The method where this alarm rule is used and where the expected value of the differentiated series is assumed to be constant, conditional on the regime, is hereafter referred to as the PHM method.

In the literature on surveillance the constants are often set so that the false alarms are under control. The most common way is to control the $A R L^{\circ}$, (the Average Run Length to the first false alarm). Another way, which is used here, is to control the MRL ${ }^{0}$, which is the median run length.

In much of the theoretical work, e.g. Shiryaev (1963) and Frisén and de Maré (1991) and especially where optimality theorems are available, the false alarms are measured by the total probability of false alarm

$$
P\left(t_{A}<\tau\right)=\sum_{i=1}^{\infty} P(\tau=i) \cdot P\left(t_{A}<i \mid D\right) .
$$


For this measure it is necessary to specify the distribution of $\tau$.

\section{Evaluation of likelihood based methods by a Monte Carlo study}

Andersson et al. (2002b) report a Monte Carlo study of the performance of the SRlin, MSR and PHM methods. The comparison was made with respect to the delay of motivated alarms and the predictive value of an alarm. The methods were made comparable by adjusting the alarm limit so that a false alarm property $\left(\mathrm{MRL}^{\circ}\right)$ is comparable. Two situations were investigated: i) when the correct parameter values were used and ii) when the regression is miss-specified. In this section a brief summary of the results from the study is made.

\subsection{Correct parametric specifications}

If the parametric model and the parameter values used by the SRlin and PHM methods are completely known then it is an advantage to use this information. As expected the SRlin method has a shorter expected delay than the MSR method.

The PHM method does not use known information on earlier turns and thus have worse properties than the SRlin method (and in some respects also than the MSR method). The conditional expected delay of a motivated alarm is highest for MSR, for very small values of $\tau$. The MSR method needs more observations to have enough evidence of a turn. After that, the delay is slightly shorter for MSR, compared to PHM. For PHM, the effect of $\tau$ is very small. The SRlin method has the shortest delay for every $\tau$.

The PHM method has more frequent false alarms at early time points, but low alarm probability later compared with that of the SRlin and especially MSR. The total probability of a false alarm, $P\left(t_{A}<\tau\right)$, is investigated for a geometric distribution with intensity $v$. When comparing the methods, MSR has the smallest false alarm probability, whereas PHM has the largest for every value of the intensity, $v$. As a result of the assumption of a geometric distribution for $\tau$, the alarms at the beginning have a great influence on $P\left(t_{A}<\tau\right)$. The large false alarm probability for PHM for all $v$ is a result of the error-spending curve of PHM, with many early alarms.

The predictive value of an alarm at time $t, \operatorname{PV}(t)=P\left(\tau \leq t \mid t_{\mathrm{A}}=t\right)$ reflects the trust you should have in an alarm. Under the assumption of a geometric distribution with intensity $v=0.1$, it is shown that the price for the high alarm probability in the first point for the PHM method is that those alarms are of little value. PV(1) is very high for MSR, as a result of the very low false alarm rate at $t=1$. The MSR method places no parametric restrictions on the turning point curve. All information about the curve comes from data. For small values of $t$ the number of observed data is very small and thus the 
data have to be very extreme in order to call an alarm. However, as $t$ increases (and the number of observations increases) the information about the curve is improved.

\subsection{Parameters miss-specified}

In practice neither the regression model nor the values of the parameters are known. If the slopes (expansion- and recession-) are estimated from a short period, then the parameters might be very wrongly specified. Both the SRlin method and the PHM method use information from previous expansion and recession phases in the alarm statistic and for parameter estimation. We have determined (by simulations) the 95\% confidence limits for the parameters when estimated by a procedure used in practice. We have then examined the effect of errors in parameters when using the confidence limit instead of the true value.

If only the slope after the turning point (the post-peak slope) is wrongly specified, then there are only minor effects on the false alarms, the delay of a motivated alarm and the predictive value of an alarm. The predictive value is very similar between the correctly specified post-peak slope and the miss-specified post-peak slope, except at $t=1$. The difference is due to the difference in the error-spending curve. When the postpeak slope is specified as being too flat, then the alarm statistic is optimized to detect a smaller change (flatter post-peak slope) and therefore the alarms are located later on. The result is few alarms at early time points, which results in a high predictive value for $t=1$. The opposite holds for a too steep post-peak slope.

However, wrong specification of also the pre-peak slope has a major influence. For incorrect specification with both slopes being too steep, the false alarm probability is very low for small $t$. This is due to the increasing difference between the true and specified states. For small $t$ this difference is small. However, as $t$ increases, so does the difference. Thus, the likelihood for the specified $D$-state decreases and therefore, the alarm probability increases. The low false alarm probability is a result of the errorspending curve with few early alarms, in contrast to using correctly specified slopes that result in many early alarms.

For small and intermediate values of $\tau$, the delay conditional on $\tau$ is longer if both slopes are incorrectly specified so that both slopes are too steep. As $\tau$ increases, the conditional delay decreases towards an asymptote, zero. Thus for the situation where both slopes are miss-specified, the resulting delay is large unless the turning point occurs very late. In contrast, the non-parametric approach, MSR, has a long delay time for extremely early turns, but quickly reaches a reasonable asymptotic value.

The conclusion is that wrong assumptions about slopes do have a major effect on the properties and that the MSR method gives a safe way to avoid this. 


\section{$4 \quad$ Using methods for turning point detection on real data}

When suggesting a new statistical method, one often wants to evaluate the method and establish its strong and weak sides. If the evaluation is made by a Monte Carlo study, it is possible to make statements about, for example, the standard errors of the evaluation measures. However, ultimately the method will be used on real data, which presents some additional problems, such as seasonal variation, presence of trend, autocorrelation and noise, how to handle the situation with several cyclical processes. In the paper by Andersson et al. (2002a), these problems are also discussed and here a brief description is given.

\subsection{Special data problems}

\subsubsection{Smoothing}

The smoothing of observations reduces the variance and hence reduces the false alarm probability. The alarm limit 0.5 is often used for the posterior probability. This will not correspond to a desired false alarm rate. Therefore one suggestion for reducing the false alarm rate is to smooth the observations. Öller (1986) and Koskinen and Öller (1998) use exponential weights. Hall et al. (1995) use smoothing by kernel estimators. However, there are also disadvantages. The smoothing will introduce an autocorrelation and reduce the distinctness of the turning point. The surveillance method EWMA (see e.g. Crowder (1987), Domangue and Patch (1991) and Frisén and Sonesson (2001)), which is designed for a controlled false alarm rate at the same time as the variability is reduced by smoothing, might be an alternative.

In a Monte Carlo study (Andersson et al. (2002a)), the effect of smoothing is investigated for the PHM method. The skewness of the density of the time of the alarm is different depending on whether the data is smoothed or not. It was shown that the reduced distinctness of the turning point, due to smoothing, decreases the probability of successful detection.

\subsubsection{Seasonality}

Leading economic indicators are often measured monthly or quarterly and thus often contain seasonal variation, which could complicate the monitoring. The seasonal variation can be considerable. Seasonality cannot be neglected in the monitoring without the risk of seriously wrong conclusions. However, when adjusting for seasonality, it is important that the structure of the original series is not disturbed and that the time of the turning point is preserved (see e.g. Ghysel and Perron (1996) and Franses and Paap (1999)). 
It is demonstrated, in Andersson and Bock (2001), that in a surveillance situation the detection is delayed when a data transformation such as differentiating or moving average is used.

\subsubsection{Autocorrelation}

Lahiri and Wang (1994) evaluate the performance of models with autocorrelation of order $r=\{0,1,2,3,4\}$. They find that the introduction of autocorrelation in the errors increases the risk of wrong inference concerning turning points. If the assumption of a time dependent stochastic term is used, when the process is in fact autocorrelated, the result is an increased false alarm probability. This effect of autocorrelation can be dealt with by adjusting the alarm limit. It is sometimes argued (see Ivanova et al. (2000)) that the effect of the autoregressive parameters will largely be captured by the probabilities of remaining in the current state. Most suggested approaches for the detection of turns in business cycles assume independent observations conditionally on the state.

\subsubsection{Trend}

Many macroeconomic variables can be characterized as cyclical movements around a trend and sometimes it is useful to adjust for the trend. Adjusting for trend implies a data transformation, which may result in a distortion of the characteristics of the original series. Gordon (1997) warns against using other information from the data than that which is directly associated with the business cycle turning points. Trend removal is discussed by Canova (1998) and Canova (1999) and one conclusion is that statements concerning the turning points are not independent of the statistical assumptions needed to extract trends.

In most HMM approaches and also the one considered here (PHM), differentiation is used as a way of adjusting for the trend. In the HMM approaches a long series is used in order to extract information about all earlier turns. However for the SRlin and MSR methods the knowledge on the last turn is utilized and thus the need for trend adjustment is less since only a short time series is analyzed.

\subsubsection{Several leading indicators}

By the common movement approach (Stock and Watson (1991) and Stock and Watson (1993)), a business cycle is characterized as the cyclical movement of many economical activities. Important information is contained in the relation between the turns of different indices. The common movements of coincident variables arise from an unobservable common factor (the overall state of the economy). Diebold and Rudebusch (1996) consider the common movements of coincident variables where the common factor depends on a hidden Markov chain with two states. Birchenhall et al. (1999) extract a business-cycle index from a vector of time series.

In Koskinen and Öller (1998) a joint vector of leading indicators with a common time of turn is monitored. When several variables have the same change point (or a 
known lag) Wessman (1998) demonstrates that the minimal sufficient alarm statistic is univariate. Thus a reduction to a univariate statistic is possible. For reviews on multivariate surveillance, see Wessman (1999) and Frisén (2002).

\subsection{Evaluation by data on the Swedish industrial production}

A common way to evaluate a method for turning point detection is to apply it on a set of real data. This is done in Andersson et al. (2002a) and here a brief summary of the results is given.

Seasonally adjusted quarterly data on the (logarithm of the) Swedish industrial production is used, from the period 1987Q2:1992Q2. For this period, according to official records (National Institute of Economic Research (1992)), the transition from expansion to recession occurs after 11 quarters (almost 3 years). All three methods (MSR, PHM and SRlin) give alarms earlier than the official times for this set of data.

SRlin and PHM use the assumption of a piecewise linear model, which fits less well since the slope is decreasing just before the turning point. McQueen and Thorley (1993) discuss this common phenomenon. If a plateau is an early indication of a coming recession, then the early alarms for the three methods can be considered to be an advantage, since they can be seen as warnings.

The model in a simulation study might not be representative of the process we want to study, whereas an actual data set certainly is representative. However, the actual data set might deviate stochastically from the process of interest. One difficulty with evaluation of the properties of a method by one sample of real data is the definition of the turning point. Another difficulty is to know whether the sample is an extreme result or typical. By using several real data sets (instead of just one) in the evaluation, the stochastic variation in the measures of evaluation would be reduced. However, these analyses must be independent (for example regarding the estimates of the parameters), in order to reduce the variance of the stochastic components.

\section{Discussion}

Three likelihood based methods (MSR, PHM and SRlin) has been presented. Differences and similarities between the methods have been pointed out.

The PHM method (contrary to the other two) does not assume knowledge of the type of the next turn, which has a major impact on the test statistic. Because this information is not used, there are frequent false alarms at early time points, but low alarm probability later. The results of this allocation of the alarm probability are short delay for alarm for early changes, but long delays for changes that occur late.

The intensity of a turning point is an important parameter. The most common assumption is that the intensity is constant and that the transition probabilities are 
constant over the cycles. A constant transition probability implies that the time of the turn has a geometric distribution, with the highest values at the early times, which is not in accordance with reality for business cycles. If the intensity is estimated from historical data, then the impact of the actual data would be reduced. It is very important that the monitoring system has the ability to detect a turning point also when this happens at an unexpected time. Thus, here we prefer to use a non-informative prior for the time of the turn (the SR approach).

Using a parametric model improves the performance of a method if the parameter information is reliable. But a wrongly specified parametric model has serious effects. The non-parametric approach, which only uses the change in monotonicity, has the advantage that it works also when reliable information on the parametric function is not available. Also important is that the non-parametric method does not assume that all expansion phases (or recession phases) have the same level and parametric shape. Thus, the very bad properties demonstrated for wrong specification of the slope give a warning. The safe way by the MSR method might be preferred.

A period of the Swedish industrial production is analyzed in Section 4, in order to compare the three methods. For this data set all three methods call an alarm before the turn, largely due to the fact that the slope is decreasing just before the transition. This is not necessarily a disadvantage. The drawback of evaluation by real data is that one is limited to one or a few replicates. Thereby it is hard to make inference about the certainty of the results.

\section{References}

Andersson, E. (2001) Turning point detection using non-parametric statistical surveillance. Evaluation of some influential factors. Research Report, Department of Statistics, Göteborg University, Sweden, 2001:3

Andersson, E. (2002) Monitoring cyclical processes - a nonparametric approach. Journal of Applied Statistics, 29.

Andersson, E. and Bock, D. (2001) On seasonal filters and monotonicity. Research Report, Department of Statistics, Göteborg University, Sweden, 2001:4

Andersson, E., Bock, D. and Frisén, M. (2002a) Some statistical aspects on methods for detection of turning points in business cycles. Research Report,

Andersson, E., Bock, D. and Frisén, M. (2002b) Statistical surveillance of cyclical processes. Detection of turning points in business cycles. Research Report,

Birchenhall, C. R., Jessen, H., Osborn, D. R. and Simpson, P. (1999) Predicting US businesscycle regimes. Journal of Business \& Economic Statistics, 17, 313-323.

Canova, F. (1998) Detrending and business cycle facts. Journal of Monetary Economics, 41, $475-512$.

Canova, F. (1999) Does detrending matter for the determination of the reference cycle and the selection of turning points? The Economic Journal, 109, 126-150.

Crowder, S. (1987) A simple method for studying run-length distributions of exponantially weighted moving average charts. Technometrics, 29, 401-407.

Diebold, F. X. and Rudebusch, G. D. (1996) Measuring business cycles: A modern perspective. Review of Economics and Statistics, 78, 67-77. 
Domangue, R. and Patch, S. C. (1991) Some omnibus exponentially weighted moving average statistical process monitoring schemes. Technometrics, 33, 299-313.

Franses, H. P. and Paap, R. (1999) Does Seasonality Influence the Dating of Business Cycle Turning Points? Journal of Macroeconomics, 21, 79-92.

Frisén, M. (1986) Unimodal regression. The Statistician, 35, 479-485.

Frisén, M. (1994) Statistical Surveillance of Business Cycles. Research Report, Department of Statistics, Göteborg University, Sweden, 1994:1 (Revised 2000)

Frisén, M. (2002) Statistical surveillance. Optimality and methods. Research Report, 2002:2 Department of Statistics, Göteborg University,

Frisén, M. and de Maré, J. (1991) Optimal Surveillance. Biometrika, 78, 271-80.

Frisén, M. and Sonesson, C. (2001) Optimal surveillance by EWMA. Research Report, Department of Statistics, Göteborg University, Sweden, 2000:7

Frisén, M. and Wessman, P. (1999) Evaluations of likelihood ratio methods for surveillance. Differences and robustness. Communications in Statistics. Simulations and Computations, 28, 597-622.

Ghysel, E. and Perron, P. (1996) The Effect of Linear Filters on Dynamic Time Series With Structural Change. Journal of Econometrics, 70, 69-97.

Gordon, S. (1997) Stochastic trends, deterministric trends, and business cycle turning points. Journal of Applied Econometrics, 12, 411-434.

Hackl, P. and Westlund, A. H. (1989) Statistical analysis of "Structural Change". Empirical Economics, 14, 167-192.

Hall, P., Marron, J. S. and Titterington, D. M. (1995) On partial local smoothing rules for curve estimation. Biometrika, 82, 575-587.

Hamilton, J. D. (1989) A new approach to the economic analysis of nonstationary time series and the business cycle. Econometrica, 57, 357-384.

Ivanova, D., Lahiri, K. and Seitz, F. (2000) Interest rate spreads as predictors of German inflation and business cycles. International Journal of Forecasting, 16, 39-58.

Jun, D. B. and Joo, Y. J. (1993) Predicting turning points in business cycles by detection of slope changes in the leading composite index. Journal of Forecasting, 12, 197-213.

Kim, C.-J. and Nelson, C. R. (1998) Business cycle turning points, a new coincident index, and tests of duration dependence based on a dynamic factor model with regime switching. Review of Economics and Statistics, 80, 188-201.

Koskinen, L. and Öller, L.-E. (1998) A Classifying Procedure for Signalling Turning Points. Research Report, National institute of economic research. Konjunkturinstitutet, Ekonomiska rådet, Stockholm, Sweden, 59

Lahiri, K. and Wang, J. G. (1994) Predicting cyclical turning points with a leading index in a Markov Switching model. Journal of Forecasting, 13, 245-263.

Lai, T. L. (1995) Sequential Changepoint Detection in Quality-Control and Dynamical-Systems. Journal of the Royal Statistical Society B, 57, 613-658.

Layton, A. P. (1996) Dating and predicting phase changes in the U.S. business cycle. International Journal of Forecasting, 12, 417-428.

Li, D. T. and Dorfman, J. H. (1996) Predicting turning points through the integration of multiple models. Journal of Business and Economic Statistics, 14, 421-428.

McQueen, G. and Thorley, S. (1993) Asymmetric business cycle turning points. Journal of Monetary Economics, 31, 341-362.

National Institute of Economic Research (1992) Konjunkturläget Maj 1992. Research Report, National Institute of Economic Research, Stockholm, Sweden,

Neftci, S. (1982) Optimal prediction of cyclical downturns. Journal of Economic Dynamics and Control, 4, 225-41.

Robertson, T., Wright, F. T. and Dykstra, R. L. (1988) Order Restricted Statistical Inference, John Wiley \& Sons Ltd.

Shiryaev, A. N. (1963) On optimum methods in quickest detection problems. Theory of Probability and its Applications., 8, 22-46. 
Srivastava, M. S. and Wu, Y. (1993) Comparison of EWMA, CUSUM and Shiryayev-Roberts Procedures for Detecting a Shift in the Mean. Annals of Statistics, 21, 645-670.

Stock, J. H. and Watson, M. W. (1991) A Probability Model of the Coincident Economic Indicators. In Leading Economic Indicators: New Approaches and Forecasting Records(Ed, Moore, G. H.) Cambridge University Press, Cambridge, pp. 63-89.

Stock, J. H. and Watson, M. W. (1993) A Procedure for Predicting Recessions With Leading Indicators: Econometric Issues and Recent Experience. In Business Cycles, Indicators and Forecasting, Vol. 28 (Ed, Watson, M. W.) University of Chicago Press, Chicago.

Wessman, P. (1998) Some Principles for surveillance adopted for multivariate processes with a common change point. Communications in Statistics. Theory and Methods, 27, 11431161.

Wessman, P. (1999) Studies on the surveillance of univariate and multivariate processes. In Department of StatisticsGöteborg University, Sweden, Göteborg, pp. 103.

Westlund, A. H. and Zackrisson, U. (1986) On the prediction of structurally varying systems. Technological Forecasting and Social Change, 30, 63-72.

Wetherhill, G. B. and Brown, D. W. (1991) Statistical Process Control: Theory and Practice, Chapman and Hill, London.

Zarnowitz, V. and Moore, G. H. (1982) Sequential signals of recessions and recovery. Journal of Business, 55, 57-85.

Zellner, A., Hong, C. and Min, C.-K. (1991) Forecasting Turning Points in International Output Growth Rates Using Bayesian Exponentially Weighted Autoregression, Time-Varying Parameter, and Pooling Techniques. Journal of Econometrics, 49, 275-304.

Öller, L.-E. (1986) A note on exponentially smoothed seasonal differences. Journal of Business and Economic Statistics, 4, 485-489. 

$\underline{\text { Research Report }}$

2001:1 Holgersson, H.E.T.: $\quad$ On assessing multivariate normality.

2001:2 Sonesson, C. \& Bock, D.:

Statistical issues in public health monitoring A review and discussion.

2001:3 Andersson, E.:

Turning point detection using non-parametric statistical surveillance. Evaluation of some influential factors.

2001:4 Andersson, E. \&

On seasonal filters and monotonicity. Bock, D.:

2001:5 Andersson, E., Bock, D. \& Frisén, M.:

Likelihood based methods for detection of turning points in business cycles.

A comparative study.

2001:6 Sonesson, C.:

Evaluations of some exponentially weighted moving average methods.

2001:7 Sonesson, C.:

Statistical surveillance.

Exponentially weighted moving average methods and public health monitoring.

2002:1 Frisén, M. \&

Sonesson, $\mathrm{C}$.:

Optimal surveillance based on exponentially weighted moving averages.

2002:2 Frisén, M.:

Statistical surveillance. Optimality and methods.

2002:3 Jonsson, R. \& Bayes prediction of binary outcomes based Persson, A.: on correlated discrete predictors.

2002:4 Persson, A.:

Prediction of work resumption among men and women with lower back- and neck pain in a Swedish population.

2002:5 Persson, A.:

Prediction of work resumption in theory and practice. 